The Astrophysical Journal, 241:L27-L31, 1980 October 1

(c) 1980. The American Astronomical Society. All rights reserved. Printed in U.S.A.

\title{
OPTICAL OBSERVATIONS OF THE ULTRAHIGH-EXCITATION WOLF-RAYET STAR SANDULEAK 3
}

\author{
MICHAEL J. BARLOW \\ Anglo-Australian Observatory; and Department of Physics and Astronomy; University College London \\ J. C. BLADES \\ Anglo-Australian Observatory \\ AND \\ D. G. HummeR ${ }^{1}$ \\ Department of Physics and Astronomy, University College London \\ Received 1980 February 19; accepted 1980 June 18
}

\begin{abstract}
We have identified recombination lines of $\mathrm{O}$ VII, $\mathrm{O}$ VIII, and $\mathrm{C} \mathrm{v}$ in the optical spectrum of an $\mathrm{O}$ VI Wolf-Rayet star, representing the first non-X-ray detection of these ions in astronomical spectra and implying excitation energies in excess of $800 \mathrm{eV}$. Rapid variations on a time scale of about $150 \mathrm{~s}$ have been observed in the profile of one of the $\mathrm{O}$ vII lines.

Subject headings: stars: emission-line - stars: Wolf-Rayet
\end{abstract}

\section{INTRODUCTION}

Smith and Aller (1969) defined the O vi sequence as a subset of the Wolf-Rayet central stars of planetary nebulae which exhibit the $\mathrm{O}$ vi $3 p-3 s$ doublet at 3811 , $3834 \AA$ as one of the strongest emission lines in their optical spectra. Sanduleak (1971) listed five O vi stars which were not apparently associated with planetary nebulae. One of these, located in the Small Magellanic Cloud, has an absolute magnitude indicative of Population I status. Apart from the strongest bands, the emission-line characteristics of these five stars have not been explored before now. We have acquired optical spectra of all five of these stars and present here data on Sanduleak's star 3 (hereafter designed Sand 3). This star, a galactic object, was chosen for a detailed study because it is unique among the five stars in possessing the narrowest and most sharply peaked emission lines, thus facilitating line identifications. The full width of the $O$ vi $\lambda \lambda 3811,3834$ doublet implies a wind expansion velocity of $2700 \mathrm{~km} \mathrm{~s}^{-1}$; compared to $5500 \mathrm{~km} \mathrm{~s}^{-1}$ for Sanduleak 4, for instance. An interesting characteristic of Sand 3 is that none of its optical emission lines, some of which are very strong, show any trace of $\mathrm{P}$ Cygni-type absorption structure. All appear to be pure emission lines. The lack of a surrounding nebula eliminates the problem of contamination by nebular lines which is often encountered in studies of $O$ vI stars in planetary nebulae. We present here our results which indicate that Sand 3 has the highest excitation of any hot star yet observed.

\footnotetext{
${ }^{1}$ On leave from the Joint Institute for Laboratory Astrophysics, National Bureau of Standards and University of Colorado, Boulder, Colorado.
}

\section{OBSERVATIONS AND LINE IDENTIFICATIONS}

All the spectra discussed here were taken with the $3.9 \mathrm{~m}$ Anglo-Australian Telescope using the RGO spectrograph with the UCL Image Photon Counting System as detector (Boksenberg and Burgess 1973). Prior to wavelength calibration all stellar spectra were divided by a flat-field exposure in order to remove pixel-to-pixel sensitivity variations in the system. Wavelength calibration was provided by means of a fifth-order polynomial fit to wavelength versus channel for typically 40 comparison arc lines, whose centroids were determined by Gaussian quadrature. The arc exposures were taken immediately before and after each object exposure and then summed to provide a mean calibration (separate tests have shown that the centroids of the arc lines are stable to better than 0.1 channels rms over periods of the order of $2000 \mathrm{~s}$ ). The dispersion used for our observations was $33 \AA \mathrm{mm}^{-1}$, yielding $0.5 \AA$ per channel (one spectrum covering the $3800 \AA$ region at $10 \AA \mathrm{mm}^{-1}$ was also obtained). The instrumental resolution, determined from the mean FWHM of comparison arc lines, ranged from 2.7 to 3.2 channels, depending on the exposure, which corresponds to a typical wavelength resolution of $1.5 \AA$. Optical spectrophotometry, obtained at lower resolution using the same system on 1978 October 8 , yielded equivalent magnitudes in the continuum of $B=14.4$ and $V=14.2$ for Sand 3 .

Figure 1 shows a montage of four spectra of the $3900 \AA$ region of Sand 3 . A strong emission feature about $40 \AA$ wide is present, centered at about $3893 \AA$. The most obvious identification of the feature at first sight would be with either $\mathrm{H}$ I $\lambda 3889.0, \mathrm{He}$ I $\lambda 3888.6$, or He II $\lambda 3887.5$. However, the strength of the feature at 
$3893 \AA$ is not consistent with any of these lines. If it $\therefore$ were $\mathrm{H} 8$ or the $16 \rightarrow 4$ member of the He II Pickering series, then there should be a stronger feature at $3970 \AA$, is corresponding to $\mathrm{H} 7$ or $\mathrm{He}$ II $14 \rightarrow 4$. Inspection of $\underset{1 \rightarrow 1}{\rightarrow \rightarrow 1}$ Figure $2 a$ shows that there is no detectable emission -1 feature at $3970 \AA$. Neither is there any detectable feature at $4026 \AA$, corresponding to $\mathrm{He}$ II $13 \rightarrow 4$. Finally, the feature does not appear to be He I, since no other lines of this atom are observed (e.g., He I $\lambda 4026)$; in particular, the $\lambda 5876$ line of $\mathrm{He} \mathrm{I}$ is definitely absent (Fig. 2b; the feature centered at $5865 \AA$ is $\mathrm{C}$ IV $13 \rightarrow 8$ ).

We identify the $\lambda 3893$ emission feature with the $8 \rightarrow 7$ recombination line of $\mathrm{O}$ vIr. Hallin et al. (1973) have determined a wavelength of $3892 \pm 3 \AA$ for this line from beam-foil spectroscopy, while Denne et al. (1980) found $3887.0 \pm 0.3 \AA$. The hydrogenic wavelength for this transition is $3887.1 \AA$; according to Edlen (1956), transitions between levels corresponding to a given pair of principal quantum numbers are strongly dominated by those with the largest $l$-values, whose wavelengths are closely predicted by hydrogenic

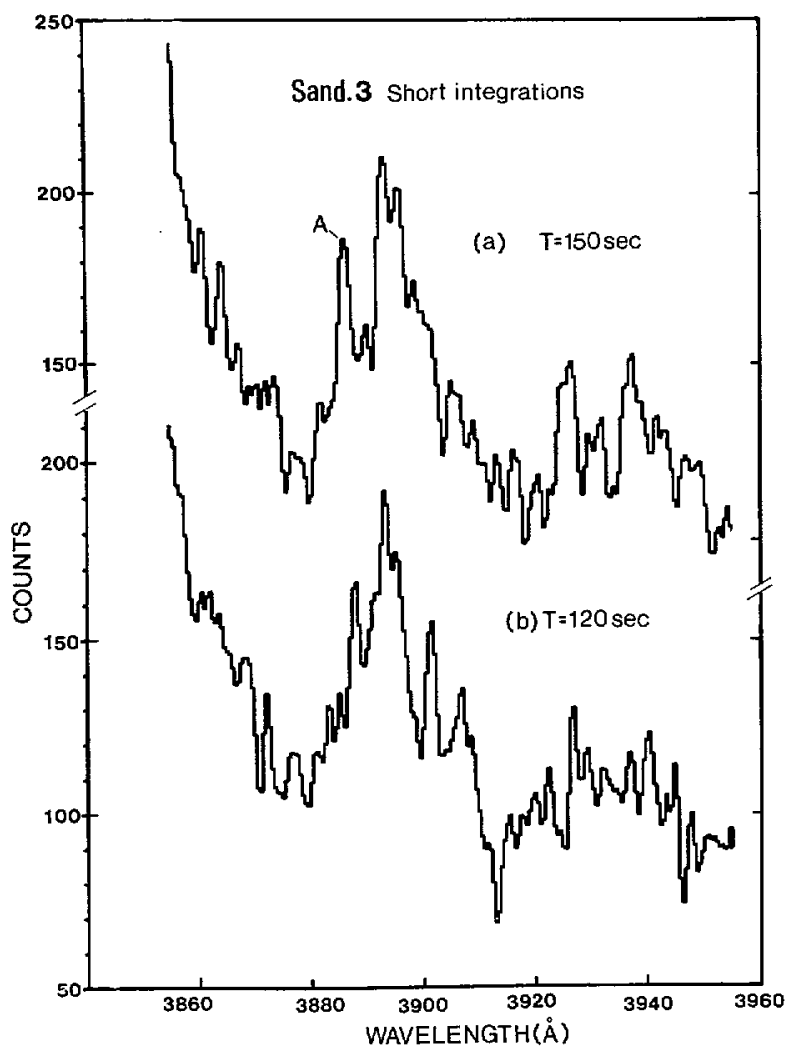

FIGs. $1 a$ and $1 b$ theory. Support for the identification of the $\lambda 3893$ feature with $\mathrm{O}$ VII comes from the presence of another emission line, centered at $5667 \AA$, in the spectrum of Sand 3 (Fig. $2 b$ ) which is attributable to the $9 \rightarrow 8$ recombination line of $\mathrm{O}$ VII (hydrogenic wavelength, $5669.7 \AA$ ). We can find no likely alternative identifications for this line. The fact that the lowest ionization stage of oxygen which we observe in Sand 3 is $\mathrm{O}$, plus the presence of many high- $n$ recombination lines of $O$ vI, lends further support to our $\mathrm{O}$ vII identifications.

The feature centered at $4860 \AA$ in Figure $2 a$ appears to be the $4859.3 \AA 8 \rightarrow 4$ Pickering line of $\mathrm{He}$ II, since its strength is consistent with that of adjacent members of the Pickering series. There appears to be no significant contribution from $\mathrm{H} \beta 4861.3 \AA$. By comparison, the sharp line centered at $4341 \AA$ in Figure $2 a$ appears too strong to be only the $10 \rightarrow 4$ line of $\mathrm{He}$ II at $4338.7 \AA$, while $\mathrm{H} \gamma$ emission appears to be ruled out by the lack of a corresponding $H \beta$ feature. We identify the feature at $4341 \AA$ with the $9 \rightarrow 8$ recombination line of $O$ VIII at $4340.5 \AA$ (Garcia and Mack 1965) with some underlying emission due to the $\mathrm{He}$ II $10 \rightarrow 4$ Pickering

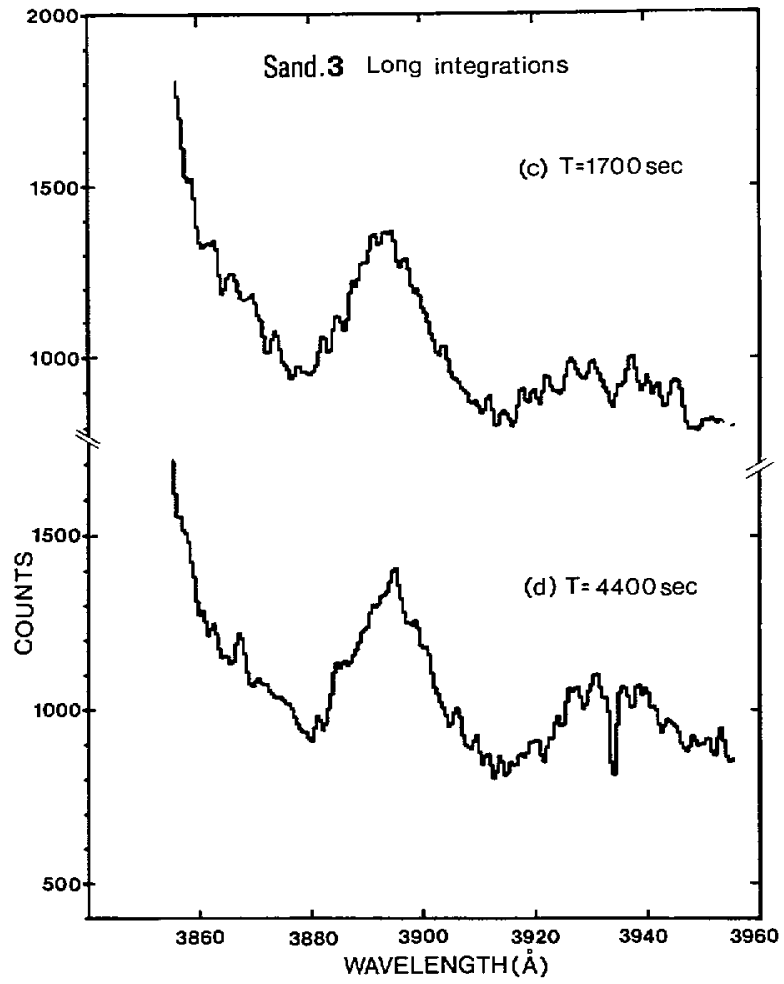

Figs. $1 c$ and $1 d$

FIG. 1.- $(a)-(d)$ The $3900 \AA$ A region of Sand 3, plotted in the form of photon counts per channel vs. wavelength, with the duration of each exposure indicated. The broad emission feature at $3893 \AA$ is identified with the $8 \rightarrow 7$ transition of $O$ vII. The wing of the extremely strong O vi $\lambda \lambda 3811,3834$ feature can be seen shortward of the $O$ vir feature. The emission feature at $3930 \AA$ is a blend of the $13 \rightarrow 7$ and $6 p \rightarrow 5 s$ transitions of $C$ IV, at $3929 \AA$ and $3935 \AA$, respectively, along with the $13 \rightarrow 9$ transition of $O$ vi at $3937 \AA$. The exposure in Fig. $1 a$ commenced at $19^{\mathrm{h}} 39^{\mathrm{m}}$ UT, $1978 \mathrm{March} 19$, with the exposure in Fig. $1 b$ beginning $180 \mathrm{~s}$ later. The exposure in Fig. $1 c$ commenced at $15^{\mathrm{h}} 16^{\mathrm{m}}$ UT, 1979 July 6 , and that in Fig. $1 d$ began at $11^{\mathrm{h}} 5^{\mathrm{m}}$ UT, 1979 July 7. 

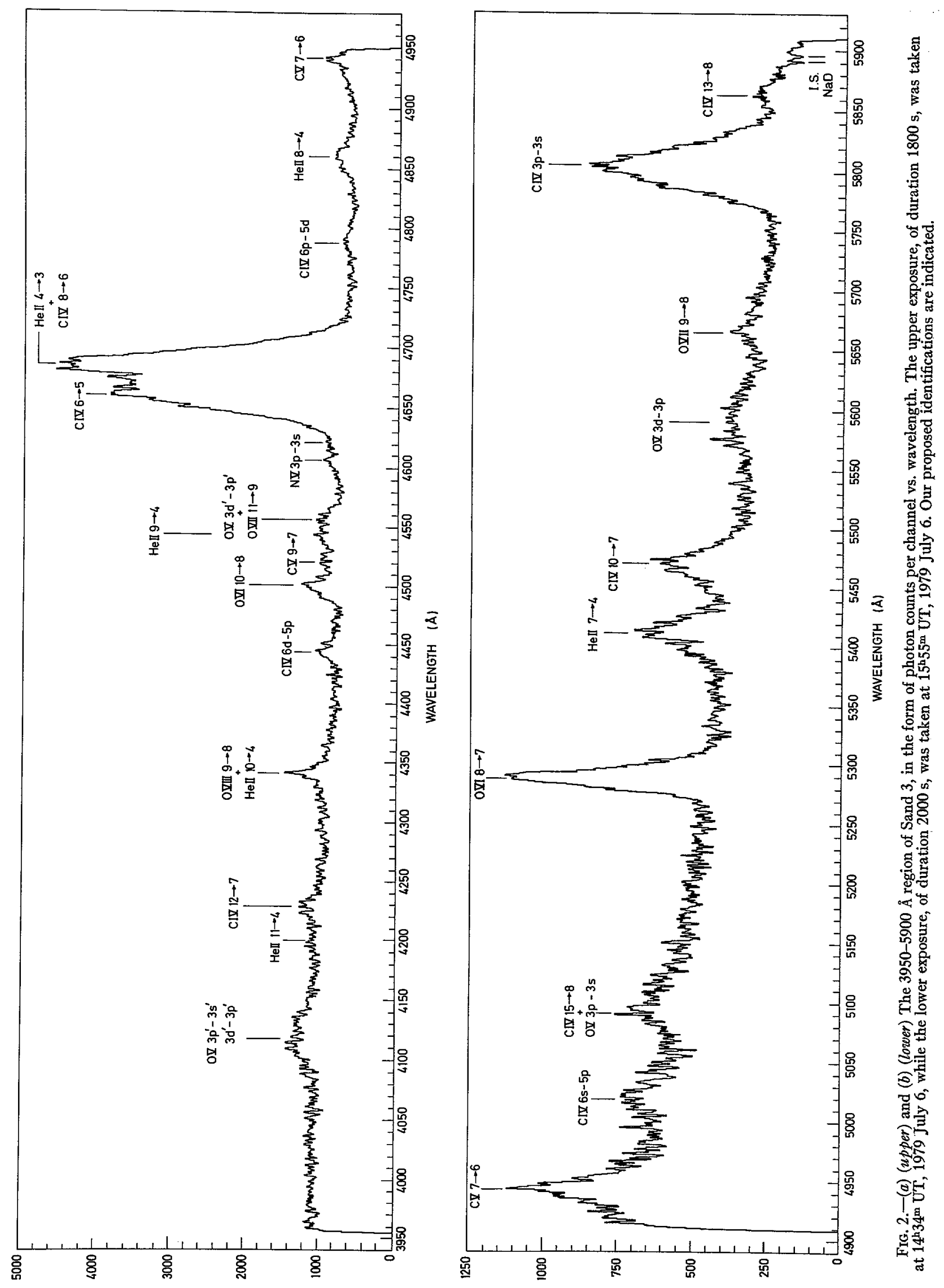
line. Since O VIII is hydrogenic, the theoretical wave$\therefore$ lengths for its transitions can be considered to be accurate. We also identify an emission feature extending from $6045 \AA$ to $6100 \AA$ in the spectrum of Sand 3 as a blend of $O$ virI $10 \rightarrow 9$ and $13 \rightarrow 11$ at 6068.2 and '-I $6064.2 \AA$, respectively, along with $O$ vII $12 \rightarrow 10$ at $6085 \AA$. There appear to be no conflicting identifications for this feature.

The strong feature centered at $4944 \AA$ in Figure $2 b$ appears to correspond to the $7 \rightarrow 6$ recombination line of $\mathrm{C} \mathrm{v}$, which has a well-determined central wavelength of $4944.7 \AA$ (Moore 1970; the hydrogenic value is $4944.9 \AA$ ). The $7 \rightarrow 6$ recombination line of $\mathrm{O} v$ has a central wavelength of $4930.3 \AA$ (Bockasten and Johansson 1968) and so does not provide an identification for the main part of the observed emission feature. The $7 \rightarrow 6$ transition of $\mathrm{N} \mathrm{v}$, on the other hand, coincides almost exactly in wavelength with the corresponding transition of $\mathrm{C} v$. However, the $3 p \rightarrow 3 s$ transition of $\mathrm{N} \mathrm{v}$ at $4604,4620 \AA$ (Fig. $2 a$ ), analogous to the very strong $3811,3834 \AA$ transition of $\mathrm{O}$ VI, is much weaker than the feature observed at $4944 \AA$, rendering the identification of the latter with $\mathrm{N} v$ extremely unlikely. The presence of $\mathrm{C} \mathrm{v}$, on the other hand, is consistent with the great strength of the C IV lines in the spectrum of Sand 3, together with the total lack of any lines due to $C$ III (witness the absence of C III $\lambda 56963 d \rightarrow 3 p$ in Fig. $2 b$ ).

The apparent noisiness of the continuum in Figure 2 does not appear to be a result of system effects such as image tube grain, since spectra taken on the same night of other early-type stars, and for which a similar number of photon counts per pixel were obtained, show much smoother continua. The spikiness apparent in the various figures is apparently caused by real and time-variable emission effects in Sand 3, which are discussed below.

Figure 1 displays four separate spectra of the O VII $\lambda 3893$ feature of Sand 3. The exposures shown in Figures $1 a$ and $1 b$, taken in 1978 April, were of duration $150 \mathrm{~s}$ and $120 \mathrm{~s}$, respectively, with the second commencing $30 \mathrm{~s}$ after the end of the first. Several distinct components are obvious in the $\mathrm{O}$ vII feature in each exposure. The strong central component is at the same velocity in each exposure, but the component at $3886 \AA$ in Figure $1 a$ (component A) has moved by $1.7 \AA$ to the red in Figure $1 b$, corresponding to a shift of $130 \mathrm{~km}$ $\mathrm{s}^{-1}$ in the $165 \mathrm{~s}$ between the midpoints of each exposure. Changes in other components are also noticeable. A shift of $1.7 \AA$ in $165 \mathrm{~s}$ would imply a line broadening of $1.6 \AA$ in $150 \mathrm{~s}$ and $1.2 \AA$ in $120 \mathrm{~s}$, provided the component moved continuously throughout both exposures. The individual components in the shorter exposure are in fact narrower than in the longer exposure, supporting the general hypothesis. Deconvolution of the measured profiles of component $A$, assuming Gaussian addition, with the instrumental resolution of $1.4 \AA$ for Figure $1 a$ and $1.35 \AA$ for Figure $1 b$ (determined from the measured half-widths of comparison arc lines), yields a line broadening of $1.6 \AA$ in Figure $1 a$ and $0.9 \AA$ in Figure $1 b$. A consequence of the shifting with time of the various velocity components in the $\lambda 3893$ feature should be that the profile becomes smoother with longer exposures, owing to the averaging of more and more individual components. Figure $1 c$ is a $1700 \mathrm{~s}$ exposure of the feature taken in 1979 July at a resolution of $1.6 \AA$. The overall profile, while still showing peaks, is noticeably smoother than the preceding two. Finally, Figure $1 d$ shows the feature in an exposure of $4400 \mathrm{~s}$ taken at $10 \AA \mathrm{mm}^{-1}$ through thin clouds in 1979 July and rebinned for comparison purposes to the same number of angstroms per channel as the other spectra. The overall spectrum is again very much smoother than in Figures $1 a$ and $1 b$.

\section{DISCUSSION}

The presence of high- $n$ transitions of $\mathrm{O}$ VII, O VIII, and $\mathrm{C} \mathrm{v}$ indicates recombination of the ions $\mathrm{O}^{+7}, \mathrm{O}^{+8}$, and $\mathrm{C}^{+5}$, respectively. The ionization potentials of $\mathrm{O}$ VI, $\mathrm{O}$ VII, and $\mathrm{O}$ vIII are 138.1, 739.3, and $871.4 \mathrm{eV}$, respectively, and that of $\mathrm{C} \mathrm{v}$ is $392.1 \mathrm{eV}$. These extremely high energies imply a coronal origin for the ionization, either directly through collisional ionization of the emitting gas or indirectly via photoionization of the emitting gas by $\mathrm{X}$-rays generated in a corona. The observations favor the photoionization model since collisional ionization would tend to set up a Boltzmann distribution of energy level populations. The presence of many high- $n$ transitions of $\mathrm{O}$ VI, $\mathrm{O}$ vII, and $\mathrm{O}$ VIII contradicts this expectation but can be explained by a model invoking photoionization followed by recombination and a cascade down the energy levels. Given that the source of the observed ionization in the wind of Sand 3 is radiative, we hypothesize that the required $\mathrm{X}$-ray flux is generated in a thin corona at the base of the atmosphere. This would be analogous to the thin corona model for $\mathrm{O}$ stars proposed by Cassinelli and Olson (1979), except that the phenomenon would appear to be much more extreme in the case of Sand 3. The moving velocity components seen in the $O$ VII emission feature are presumably generated by pulsational-type waves propagating through the atmosphere, and it seems natural to assume that the mechanism that generates these waves is also responsible for the presence of the corona.

WN-type and WC-type Wolf-Rayet stars, respectively, are thought to represent the early and late stages of the core helium-burning phase during the evolution of high-mass stars (Wills and Wilson 1978). The characteristic pulsational period of model helium-burning stars is of the order of 30 minutes (Strothers and Simon 1970), which would appear to be too long to be associated with the observed behavior of Sand 3 . Models of carbon-burning stars, on the other hand, have characteristic pulsational periods of tens of seconds upward (Mariska and Hansen 1972), just the right order of magnitude to be associated with the moving components in the $\mathrm{O}$ vII feature. In the early stages of core helium burning, carbon should be the main fusion product, but as helium is depleted the abundance of oxygen should increase, to approach that of carbon at the onset of core carbon burning (Paczyński 1973). 
Subsequent mass loss during the carbon-burning phase $\therefore$ could then reveal the processed material from the previous phase. Lines of oxygen in the spectrum of Sand 3 are in fact much more prominent relative to II those of carbon than in classical WC-type stars. Stars - such as Sanduleak 3 may therefore represent the next stage beyond the WC type in the evolution of WolfRayet stars toward their ultimate fate.
We thank Dr. K. C. Freeman for the donation of a portion of his observing time and Dr. A. J. Willis for discussions. The work of D. G. H. was supported by the Science Research Council, by the National Science Foundation through grant AST 77-23183, and by the National Bureau of Standards. Observing time on the Anglo-Australian Telescope was allocated by the United Kingdom P.A.T.T.

\section{REFERENCES}

Bockasten, K., and Johansson, K. B. 1968, Arkiv Fysik, 38, 563.

Boksenberg, A., and Burgess, D. E. 1973, in Proc. Symposium on Astronomical Observations with Television Type Sensors, ed. J. W. Glaspey and G. A. H. Walker (U. B. C., Canada: Institute of Astronomy and Space Science), p. 21.

Cassinelli, J. P., and Olson, G. L. 1979, A p. J., 229, 304.

Denne, B., Engstrom, L., Huldt, S., Ekberg, J. O., Curtis, L. J., Ishii, K., Veje, E., and Martinson, I. 1980, Phys. Scripta, 21, 151.

Edlen, B. 1956, Vistas in Astronomy, 2, 1456.

Garcia, J. D., and Mack, J. E. 1965, J. Opt. Soc. Amer., 55, 654.

Hallin, R., Lindskog, J., Marelius, A., Pihl, J., and Sjodin, R. 1973, Phys. Scripta, 8, 209.
Mariska, J. T., and Hansen, C. J. 1972, Ap. J., 171, 317.

Moore, C. E. 1970, Selected Tables of Atomic Spectra, NSRDSNBS 3, sec. 3.

Paczyński, B. 1973, in IAU Symposium 49, Wolf-Rayet and HighTemperature Stars, ed. M. K. V. Bappu and J. D. Sahade (Dordrecht: Reidel), p. 158.

Sanduleak, N. 1971, Ap. J. (Letters), 164, L71.

Smith, L. F., and Aller, L. F. 1969, Ap. J., 157, 1245.

Stothers, R., and Simon, N. R. 1970, Ap. J., 160, 1019.

Willis, A. J., and Wilson, R. 1978, M.N.R.A.S., 182, 559.

MiCHAEL J. BARLOw: Department of Physics and Astronomy, University College London, Gower Street, London WC1E 6BT, England

J. C. Blades: Anglo-Australian Observatory, P.O. Box 296, Epping, N.S.W. 2121, Australia.

D. G. Hummer: Joint Institute for Laboratory Astrophysics, University of Colorado, Boulder, CO 80309 
